\title{
Electric-field effects on finite-length superlattices
}

\author{
O. Gülseren and S. Ciraci \\ Department of Physics, Bilkent University, Bilkent 06533, Ankara, Turkey \\ (Received 23 April 1992)
}

\begin{abstract}
In this paper, we study the Wannier-Stark ladder by carrying out numerical calculations on a multiple-quantum-well structure under an applied electric field. The variation in the Wannier-Starkladder energies and the degree of localization of the corresponding wave function are examined over a wide range of values of the applied electric field. Our results show that the Wannier-Stark ladder does exist for a finite, but periodic system that consists of a large number of quantum wells having a multipleminiband structure.
\end{abstract}

Periodic structures under an electric field display interesting properties. The application of a constant electric field, $\mathbf{F}$, leads to a linear contribution, $e \mathbf{F} \cdot \mathbf{r}$, to the periodic potential of the time-independent Schrödinger equation. The resulting energy spectrum was predicted to be evenly spaced and is called a Wannier-Stark ladder (WSL)., ${ }^{1,2}$ The corresponding wave functions show Wannier-Stark localization. Another well-known feature of an external electric field applied to a semiconductor is the Franz-Keldysh effect, ${ }^{3}$ which is the formation of a low-energy tail below the band gap in the optical absorption coefficient. Later it was shown by Callaway that the electric field gives rise also to an oscillatory behavior in the optical absorption coefficient above the band gap. ${ }^{4}$ Bloch oscillation ${ }^{5}$ (which is the periodic motion of an electron in ordinary space due to reflections at the Brillouin-zone boundary) is conjectured to be an important consequence of the electric field in the periodic structure. Because of the presence of an applied constant electric field, the bands are inclined in ordinary space, making possible interband, or Zener, tunneling. ${ }^{6}$ The tunneling matrix element itself shows an oscillatory behavior with the presence of the electric field as a result of Wannier-Stark quantization. ${ }^{7}$

The dynamics of electrons in an electric field has been discussed by Wannier, ${ }^{1,2}$ who solved the time-dependent Schrödinger equation in terms of Houston's function. ${ }^{8}$ The usual treatment of this problem starts with putting the Schrödinger equation in crystal-momentum representation. $^{6}$ Inserting the wave function, which has been expanded in terms of Bloch functions, $\psi(\mathbf{r})=\sum_{n, \mathbf{k}} a_{n}(\mathbf{k}) \psi_{n \mathbf{k}}(\mathbf{r})$, into the Schrödinger equation and then projecting out the space coordinates, one obtains

$$
\left[E_{n}(\mathbf{k})+i e F \frac{\partial}{\partial k_{z}}-\epsilon\right] a_{n}(\mathbf{k})+\sum_{m} e F X_{n m}(\mathbf{k}) a_{m}(\mathbf{k})=0
$$

in the presence of a finite and constant electric field $F$ along the $z$ direction. Here $n$ denotes the band index, $E_{n}(\mathbf{k})$ is the band energy of an electron in the periodic lattice with eigenfunctions $\psi_{n \mathbf{k}}(\mathbf{r})$, and $\epsilon$ is the energy in the presence of the electric field $F$. The band coupling
$X_{n m}$ is proportional to the integral $\int_{\Omega_{0}}^{n m} d \mathbf{r} u_{n \mathbf{k}}^{*}(\mathbf{r}) \partial u_{m \mathbf{k}}(\mathbf{r}) / \partial k_{z}$ between the periodic part of the Bloch function over the volume of the unit cell, $\Omega_{0}$. If the interband coupling terms $X_{n m}$ with $n \neq m$ are neglected, one can readily obtain the solution for a given band $n$ by integration,

$$
\begin{aligned}
& a_{n}(\mathbf{k})=\sqrt{a / 2 \pi} \exp \int-\frac{i}{e F} \int_{0}^{k}\left[\epsilon-E_{n}\left(\mathbf{k}^{\prime}\right)\right. \\
& \left.\left.-e F X_{n n}\left(\mathbf{k}^{\prime}\right)\right] d k_{z}^{\prime}\right] .
\end{aligned}
$$

The periodicity requirement on $a_{n}(\mathbf{k})$ results in the WSL,

$$
\epsilon_{j, n}=j a e F+\frac{a}{2 \pi} \int_{0}^{2 \pi / a}\left[E_{n}(\mathbf{k})-e F X_{n n}(\mathbf{k})\right] d k
$$

where $a$ is the periodicity of the crystal along the field direction, and $j$ is an integer which labels the energy in Eq. (1). In a one-band system, the index $n$ of $\epsilon_{j, n}$ may be dropped. Having derived the tight-binding eigenvalues and eigenfunctions of the WSL within the one-band model, Saitoh ${ }^{9}$ discussed the validity of neglecting the interband terms.

The WSL problem has been a subject of dispute for several decades. The mathematical difficulties in handling the electric potential have been the primary cause of this lack of consensus. First of all, since the electric potential is linear in the space coordinate, it destroys the periodicity of the crystal potential. Second, the potential energy becomes unbounded for an infinite crystal, and so the states become metastable resonance states. By solving the Schrödinger equation in the $k q$ representation, Zak found a set of levels, $E+$ jaeF ${ }^{10}$ However, he argued that no WSL emerges from the continuous spectrum of $E$ corresponding to an infinite system. Within the same context, he also questioned Wannier's original equation. ${ }^{11,12}$ Another subject of controversy has been the assumption of neglecting the interband terms $X_{n m}$. Zak questioned the validity of this assumption by showing the inconsistency in the periodicity requirement of the periodic part of Bloch functions. ${ }^{11}$ Fukuyama, Bari, and Fogedby ${ }^{13}$ have studied the two-band tight-binding model, and found two interpenetrating WSL's. Their re- 
sult was also confirmed by Leo and MacKinnon, who presented a numerical solution of the two-band tightbinding Hamiltonian. ${ }^{14}$ Similar, a recent solution by Zhao of the two-band tight-binding model based on perturbation theory in the presence of weak interband coupling also resulted in two interpenetrating WSL's. ${ }^{15} \mathrm{Ra}$ binovitch, ${ }^{16}$ on the other hand, showed that a Born-von Kármán type of periodic boundary conditions is incompatible with the Schrödinger equation because of the nonperiodicity of the potential. He concluded that the WSL does not exist. The same problem has been treated numerically by Rabinovitch and Zak for a Mathieu-type model potential. ${ }^{17}$ This work also indicated the absence of the WSL.

In recent years, various other ideas have developed along with the renewal of interest in a solution of the WSL problem. Krieger and Iafrate used a vector potential to describe the electric field instead of a scalar potential. ${ }^{18,19}$ The difficulty with the nonperiodicity of Hamiltonian was thus removed, and the solution of the timedependent Schrödinger equation became equivalent to the Houston result ${ }^{8}$ obtained with the use of a scalar potential. This equivalence was also shown ${ }^{19}$ by employing a gauge transformation. A selection rule for optical transitions was derived without assuming the WSL, showing that the energy spacing is equal to the energy spacing of the WSL. However, $\mathrm{Zak}^{20}$ pointed out that the use of the vector potential to describe the electric field only shifts the problem from the space domain to the time domain. Consequently the basis set is periodic in time at lattice sites. ${ }^{20}$ In a different approach by Emin and Hart, the potential energy due to the electric field was written as a sum of a periodic sawtoothed potential and a steplike potential $^{21-23}$ whereby interband matrix elements of the steplike potential vanish. ${ }^{24}$ This approach-specifically whether the interband matrix elements of steplike potential vanish - was also questioned. ${ }^{14,25-28}$

In the experiments on the WSL of Koss and Lambert, ${ }^{29}$ the required electric field is usually too high, thus creating certain difficulties. However, localization can be observed at relatively reduced field values in superlattices, since the width of the miniband is reduced as a result of the large periodicity. In fact, recently Mendez, Agulló-Rueda, and Hong ${ }^{30}$ observed a WSL in $\mathrm{GaAs} / \mathrm{Ga}_{1-x} \mathrm{Al}_{x} \mathrm{As}$ superlattices by photocurrent and photoluminescence measurements. At about the same time, a tight-binding calculation of optical absorption coefficient by Bleuse, Bastard, and Voisin showed the field-induced localization. ${ }^{31}$ Quantum coherence was also studied in optical measurements by examining the wavefunction extensions of electrons as a function of the electric field. ${ }^{32}$ The electronic structure of finite-length superlattices under an external electric field was solved numerically, showing localization and band-mixing effects. ${ }^{33}$ Wannier-Stark localization has been found to exist not only in the electronic properties but also in the transport of electrons. ${ }^{34-39}$

Briefly, although the dispute on WSL continues, recent studies in finite systems provided evidence that the localization does exist. ${ }^{30,32,36,37,40}$ In this paper, we study further the WSL problem by solving the Schrödinger equa- tion of finite-length superlattices under a constant electric field. Based on our results obtained by numerical calculations, we present some aspects of WSL from a different perspective. The system we dealt with is an artificial structure which can be realized experimentally and the physical parameters of which can be varied to test these controversial issues. For example, the relatively larger periodicity along the growth direction of the multiplequantum-well structure, $a$, means that the difference between consecutive ladder energies, $a e F$, increases. Hence the localization can be realized and analyzed by changing the periodicity. By varying the height of the well $V_{w}$, one- and multiple-band effects on the WSL can be explored.

The system that we studied consists of $N$ wells and $N-1$ barriers, and buffer layers on both sides. In addition to the number of wells $N$, the width $w$, the depth $V_{w}$ of quantum well, the width of the barrier $b$, the thickness of the buffer layers $t$, and the effective mass $m^{*}$ of electrons are the parameters relevant to the system under consideration. Its total length is $L=d+2 t$, with $d=N w+(N-1) b$, and its local periodicity is $a=w+b$ (see Fig. 1). The one-dimensional (1D) equation of the envelope function in the presence of a finite, nonzero electric field $F$ can be written in the effective-mass approximation:

$$
\left(-\frac{\hbar^{2}}{2 m^{*}} \frac{d^{2}}{d z^{2}}+V(z)-e F z\right) \psi(z)=\epsilon \psi(z) .
$$

Here the electric field is along the growth direction $z$, and $V(z)$ is the quantum-well potential. $z$ is set to zero at the center of the multiple-quantum-well structure. The wave function vanishes at the end points due to the infinite well potential [i.e., $\psi(-L / 2)=0$ and $\psi(L / 2)=0$ ]. By using the three-point derivative formula, the 1D Schrödinger equation is transformed into a set of coupled linear equations. The convergence tests are performed by varying

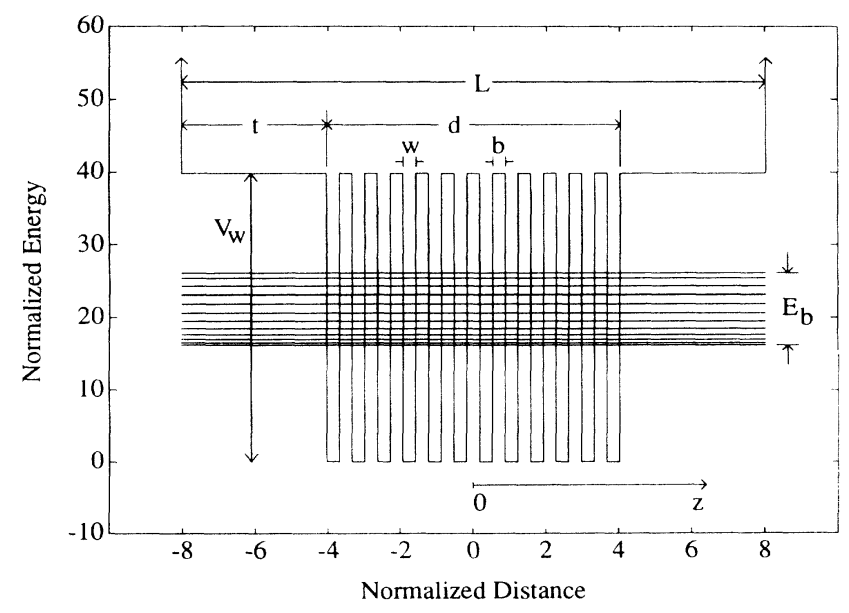

FIG. 1. Potential energy diagram for a finite periodic system consisting of $N=12$ quantum wells. The geometrical parameters of the system are $w=b=35 \AA$, and $t=400 \AA$. $z$ is set to zero at the center of the structure. The potential is taken to be infinite outside the system. 
the increment $\Delta z$ in the derivative formula from 7 to $0.03125 \AA$, and the length of the buffer layers from 25 to $5000 \AA$. It is found that $\Delta z=0.5 \AA$ and $t=400 \AA$ are appropriate for purposes of the present study. The physical quantities are given in terms of normalized units (normalized energy unit is $5.72 \mathrm{meV}$, distance unit is $100 \AA$, and electric field unit is $5.72 \mathrm{kV} / \mathrm{cm}$ for GaAs). For $F=0$, twelve states with $E<V_{w}$ form a miniband. The "continuum states" (the spectrum of which is actually discrete for finite $L$, but becomes continuous as $L \rightarrow \infty$ ) appear above $V(z)>V_{w}$.

The variation of the energy spectrum and the wavefunction amplitudes of the corresponding states as a function of electric field are shown in Fig. 2. In this fan diagram, two kinds of energy state are identified according to the dispersion of the curves. While the lower half of the well states shift down in energy, the upper half of the well states shift up in energy, with increasing electric field. The energies of the continuum states below the right-hand-side buffer-layer potential have negative dispersions. These two kinds of energy state, i.e., well states and continuum states, show a number of anticrossings for nonzero electric-field values, as seen in Fig. 2(a). This is consistent with the fact that the solutions of the 1D Schrödinger equation cannot be degenerate. The character of the well and continuum states are analyzed in terms of the absolute squares of their wave functions, $\left|\psi_{j}\right|^{2}$ in Figs. 2(b) and 2(c). The well states are extended over the periodic potential, but they become localized when $F \neq 0$. The extent of their localization narrows with increasing $F$, and eventually becomes confined to a welldefined quantum well. The continuum states falling below the potential energy of the right-hand-side buffer layer preserve their extended character in the left-handside buffer, but decay in the periodic region.

The field dependence of the localization is further analyzed by integrating the absolute square of the wave function of a certain state. The value of the integral
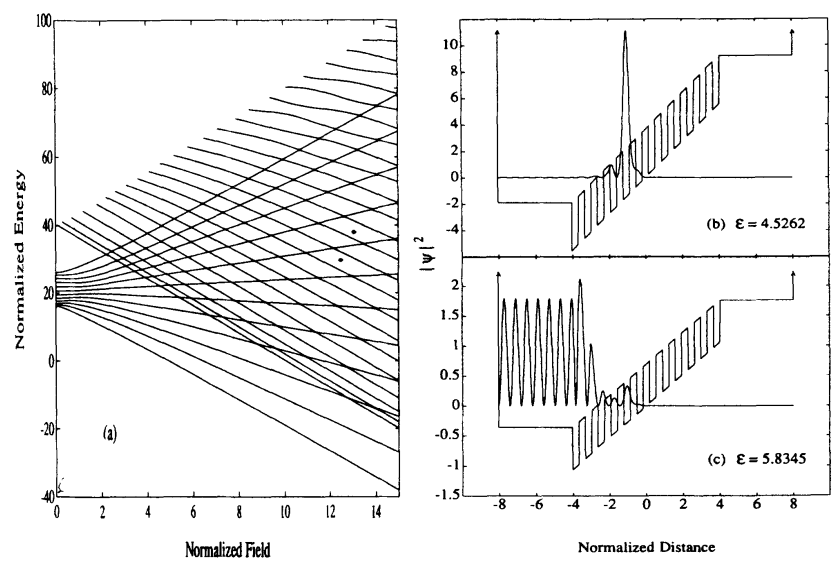

FIG. 2. (a) The fan diagram for the system described in Fig. 1 (field increment is 0.1). The squared amplitude of the wave function (multiplied by 1000) for the state confined to the fifth well (b), and for the continuum state (c) at the normalized field $F=15$.
$W_{j, m}=\int\left|\psi_{j}(z)\right|^{2} d z$ in the $m$ th well is taken as the degree of localization of state $j$ as shown in Fig. 3. The potential energy of each well shifts by $\pm a e F$ under the applied electric field $F$. For small $F$, the energy of a particular state $j$, localized in the well $m$, can overlap with the band of other well states. Hence, this state $j$ can evolve from the well $m$ to the adjacent wells within a finite time interval. Nevertheless the resonance character of $\psi_{j}$ ceases beyond a threshold field $F_{R}>E_{b} / a e$, and the localization in the well $\boldsymbol{m}$ becomes complete. For certain values of $F$ $\left(F>F_{R}\right)$, the localization in the well $m, W_{j, m}$, decreases momentarily while the weight in the buffer region increases. These points correspond to the anticrossing of continuum states with the well states as illustrated in Fig. 2(a). The anticrossing states are close in energy and hence they hybridize; both have a mixed character, being neither localized nor extended (see Fig. 4).

The degree of localization, $W_{j, m}$, which initially increases with increasing field, passes through a maximum and eventually decreases as the value of $F$ continues to increase. This behavior occurs since the top of the rectangular barrier changes into a triangular barrier under the applied electric field. The larger the field, the sharper is its apex. Beyond a critical field, the localization of the state decreases with decreasing width of the left-handside barrier.

Another aspect of the WSL examined was the detailed variation of the energy level $\epsilon_{j}(F)$ with the field. As seen in Fig. 2(a), the energy of the WSL varies first nonlinearly for small $F$. This variation becomes linear only for large $F$. This behavior can be understood by evaluating the integral

$$
\epsilon_{j}=\int_{-L / 2}^{L / 2} \psi_{j}^{*}(z)\left(H_{0}+H_{1}\right) \psi_{j}(z) d z
$$

Here $H_{0}$ is the periodic part of the Hamiltonian and $H_{1}$ is equal to $-e F z$ in the range $-d / 2<z<d / 2$, but constant elsewhere. The field dependence of a particular lev-

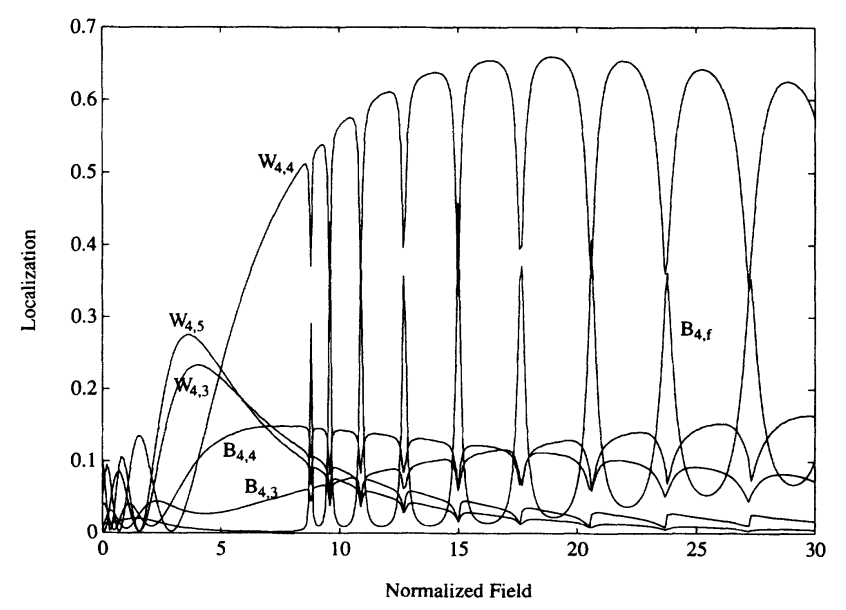

FIG. 3. Localization of the fourth well state as a function of normalized electric field. $W_{j, m}$ denotes the weight of $\left|\psi_{j}\right|^{2}$ in the $m$ th well, and $B_{j, m}, B_{j, f}$ are the weight in the $m$ th barrier and left-hand-side buffer regions, respectively. 


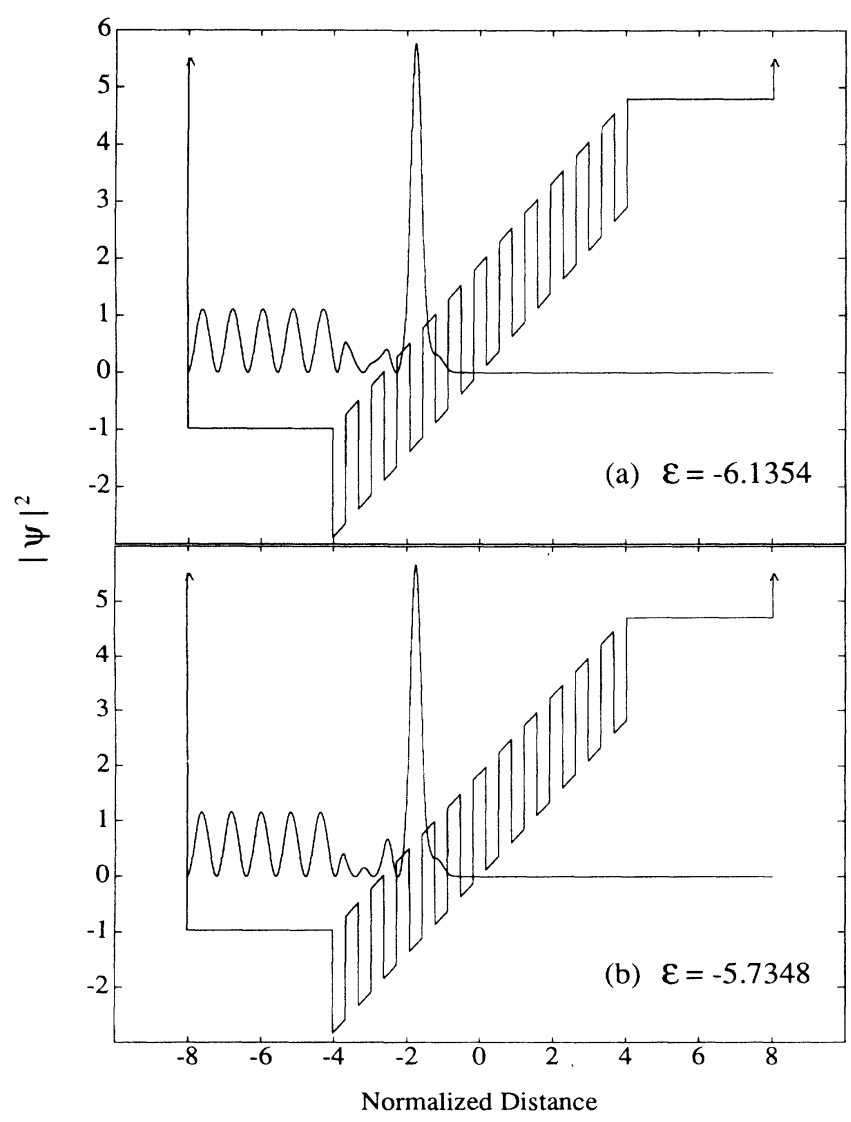

FIG. 4. $|\psi|^{2}$ (multiplied by 1000 ) vs $z$ for a continuum state and the well state $(j=4)$ at the point of anticrossing.

el of the WSL can be obtained by using the HellmannFeynman theorem

$$
\frac{\partial \epsilon_{j}}{\partial F}=\int_{-L / 2}^{L / 2} \psi_{j}^{*}(z) \frac{d H_{1}}{d F} \psi_{j}(z) d z .
$$

In view of the variation of $H_{1}$ in Fig. 2(b), the right-hand side of the above equation can be written as

$$
\begin{aligned}
\frac{\partial \epsilon_{j}}{\partial F}= & -\frac{d}{2} e \int_{-L / 2}^{-d / 2} \psi_{j}^{*}(z) \psi_{j}(z) d z+e \int_{-d / 2}^{d / 2} z \psi_{j}^{*}(z) \psi_{j}(z) d z \\
& +\frac{d}{2} e \int_{d / 2}^{L / 2} \psi_{j}^{*}(z) \psi_{j}(z) d z
\end{aligned}
$$

For small values of electric field, the well states have significant spatial extent; their energies display nonlinear shifts according to the values of integral in Eq. (7). However, the confinement to individual wells is enhanced with increasing $F$, and it is achieved to great extent for $F$ greater than $F_{R}$, but less than the value beyond which the transmission to the buffer layer begins to increase. This causes the first and third integrals in Eq. (7) to vanish, but the expectation value of the position in the second integral to be equal to the position of the center of the confining well. As a result, the energies of well states can be expressed as

$$
\epsilon_{j}=\bar{E} \pm\left\{\begin{array}{l}
j a e F, \quad j=0,1,2, \ldots, \frac{N-1}{2} \text { for odd } N \\
\left(j-\frac{1}{2}\right) a e F, \quad j=1,2,3, \ldots, \frac{N}{2} \text { for even } N,
\end{array}\right.
$$

where $\bar{E}$ is the average band energy. The calculated slopes from the fan diagram seen in Fig. 2(a) are in good agreement with Eq. (8). For the low-lying continuum states, the wave function is only extended in the lefthand-side buffer layer, and their extension in the superlattice region increases with increasing energy. They are extended through the whole system for very large energies. Hence, first term of Eq. (7) is dominant for low-lying states in energy, while all of the three terms contribute for high-energy states. Therefore, the slope of the energy versus the electric field is approximately $-d e / 2$ for the lowest-lying state, but is zero for very-high-energy states.

The effects of the finite buffer layers on both sides of the multiple-quantum-well structure are examined by varying their extent. The continuum states are discrete because of the finite size of the system but their number increases with increasing $t$, and eventually they become quasicontinuous for $t \rightarrow \infty$. The number of anticrossings with WSL states in the fan diagram increases with increasing $t$ as seen in Fig. 5. The degree of localization of the well states, $W_{j, m}$, decreases if $\epsilon_{j}$ is above the potential of the left-hand-side buffer layer $\left[V_{0}=V_{w}-(d / 2) e F\right]$ because of the small sinusoidal tail of $\psi_{j}$ for $-L / 2<z<-d / 2$. In contrast to that the localization of the well states is unaltered if their energies lie below $V_{0}$ because of the exponentially decaying tail of the wave function in the buffer region. The electric-field values for which the state $j$ lies below the $V_{0}$ is given by

$$
F_{c}=\frac{V_{w}-\bar{E}}{e\left[\frac{w}{2}+(j-1)(w+b)\right]} .
$$

The comparison of the $F_{c}$ calculated from Eq. (9) with the localization curves shows full agreement.

The number of quantum wells, $N$, is a crucial parame-

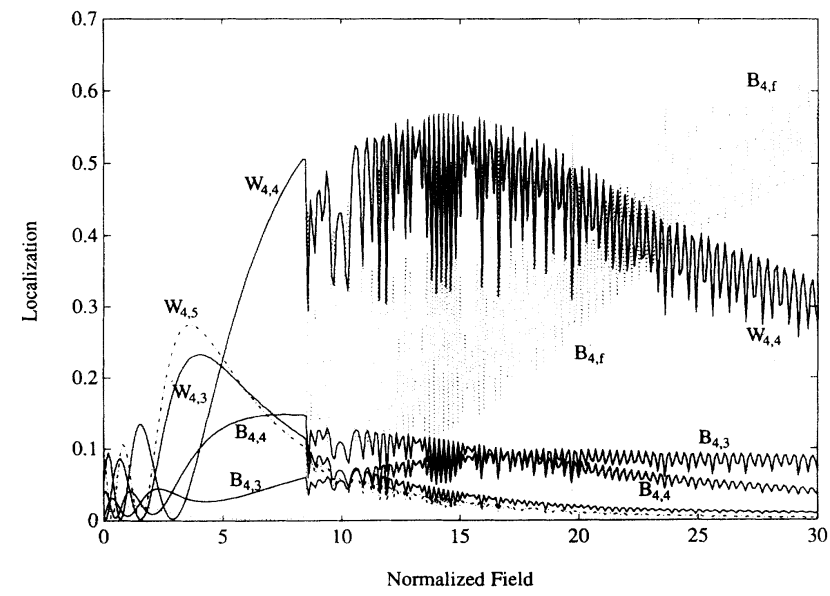

FIG. 5. Localization of the well state $(j=4)$ vs the electric field $F$ for $t=5000 \AA$. 


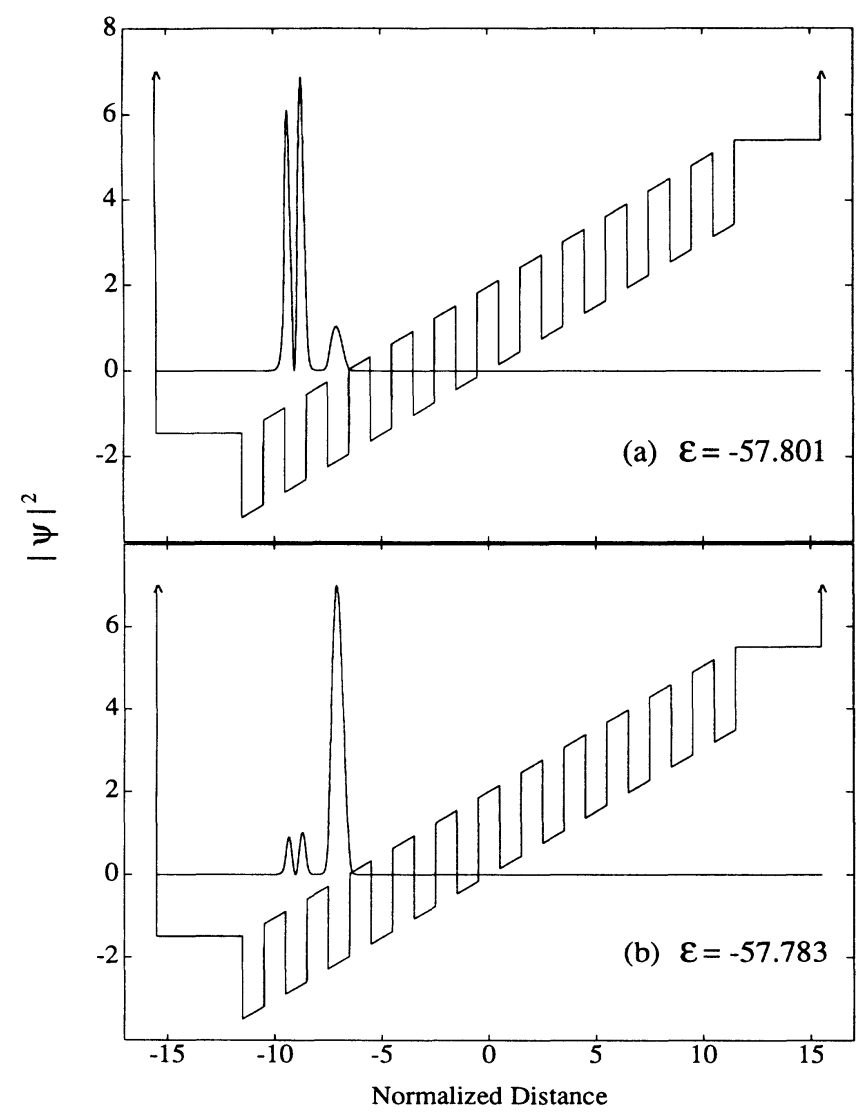

FIG. 6. $|\psi|^{2}$ (multiplied by 1000 ) vs $z$ for the well state $(j=2)$ of the second band (a), and the well state $(j=3)$ of the first band at the point of anticrossing at $F=9.1$.

ter for the WSL and has been at the center of dispute. As $N \rightarrow \infty$, the states forming the miniband become continuous and the WSL is destroyed, according to some earlier works. $^{10}$ Here we address this issue and examine the changes in the WSL spectrum as a result of increasing $N$. The width of the miniband $E_{b}$ is practically unaffected, but new discrete states appear within the miniband as a result of increasing $N$. According to Eq. (8) the slope of the WSL states changes with increasing $N$. At the same time, the slopes of the continuum states in the fan diagram also change since the extent of the whole system, $L$, increases with increasing $N$. These two changes compensate each other, and the anticrossings occur at the same electric field value, and hence localization curves such as those in Fig. 3 become independent of $N$, apart from the boundary effects. Even if these results imply that WSL exists in the limit of $N \rightarrow \infty$, the consecutive anticrossings appear to join. This certainly reduces the localization.

The number of minibands is increased by increasing $w$ and $V_{w}$. For example, for $w=100 \AA$ and $V_{w}=344 \mathrm{meV}$, three minibands $(n=1,2,3)$ occur in the multiplequantum-well structure. Most of the previous arguments in this study apply to the multiple-miniband case. In addition to the anticrossing with continuum states, states of different minibands anticross because of different slopes resulting in the interband mixing and slight delocalization consistent with some recent experimental studies. ${ }^{40}$ The absolute squares of the two wave functions which engage in such an anticrossing are illustrated in Fig. 6. The nodal structure of $\left|\psi_{j}\right|^{2}$ identifies the band to which state $j$ belongs. The interband mixing is evident, with the secondary peak located near the major peak. The nodal structure of this secondary peak also identifies the character of the other band involved in the mixing.

The important findings of this study are summarized as follows: (i) In a finite system consisting of a periodic multiple-quantum-well structure with a buffer layer at each end, WSL forms and, beyond a threshold field, states are confined to individual wells. Discrete energies of the states forming a miniband vary first nonlinearly, then linearly with increasing field. As the external electric field increases, the degree of localization of the WSL state increases, passes through a maximum, and eventually starts to decrease. (ii) The character of states are mixed at the point of anticrossing. (iii) The present results, which are obtained from numerical calculations, show that WSL does exist in the multiple-quantum-well structure including a large number of wells and multipleminiband structure.
${ }^{1}$ G. H. Wannier, Phys. Rev. 117, 432 (1960).

${ }^{2}$ G. H. Wannier, Rev. Mod. Phys. 34, 645 (1962).

${ }^{3}$ W. Franz, Z. Naturforsch. 13a, 484 (1958); L. V. Keldysh, Zh. Eksp. Teor. Fiz. 34, 1138 (1958) [Sov. Phys. JETP 7, 788 (1958)].

4J. Callaway, Phys. Rev. 130, 549 (1963).

${ }^{5}$ B. K. Ridley, Quantum Processes in Semiconductors (Oxford Science Publications, Oxford, 1988), pp. 50-53.

${ }^{6}$ E. O. Kane, J. Phys. Chem. Solids 12, 181 (1959).

${ }^{7}$ P. N. Argyres, Phys. Rev. 126, 1386 (1962).

${ }^{8}$ W. V. Houston, Phys. Rev. 57, 184 (1940).

${ }^{9}$ M. Saitoh, J. Phys. C 5, 914 (1972).

10J. Zak, Phys. Rev. 168, 686 (1968); in Solid State Physics, edited by F. Seitz, D. Turnbull, and H. Ehrenreich (Academic, New York, 1972), Vol. 27, pp. 1-61.

${ }^{11}$ J. Zak, Phys. Rev. Lett. 20, 1477 (1968).
${ }^{12}$ G. H. Wannier, Phys. Rev. 181, 1364 (1969); J. Zak, ibid. 181, 1366 (1969).

${ }^{13}$ H. Fukuyama, R. A. Bari, and H. C. Fogedby, Phys. Rev. B 8, 5579 (1973).

14J. Leo and A. MacKinnon, J. Phys. Condens. Matter 1, 1449 (1989).

${ }^{15}$ X. Zhao, Phys. Lett. A 154, 275 (1991).

${ }^{16}$ A. Rabinovitch, Phys. Lett. 33A, 403 (1970).

${ }^{17}$ A. Rabinovitch and J. Zak, Phys. Rev. B 4, 2358 (1971).

${ }^{18}$ C. Kittel, Quantum Theory of Solids (Wiley, New York, 1963), p. 190.

19J. B. Krieger and G. J. Iafrate, Phys. Rev. B 33, 5494 (1986).

${ }^{20}$ J. Zak, Phys. Rev. B 38, 6322 (1988); J. B. Krieger and G. J. Iafrate, ibid. 38, 6324 (1988).

${ }^{21}$ D. Emin and C. F. Hart, Phys. Rev. B 36, 7353 (1987).

${ }^{22}$ C. F. Hart, Phys. Rev. B 38, 2158 (1988). 
${ }^{23}$ C. F. Hart and D. Emin, Phys. Rev. B 37, 6100 (1988).

24J. Zak, Phys. Lett. 76A, 287 (1980).

${ }^{25}$ P. N. Argyres and S. Sfiat, Phys. Lett. A 145, 451 (1990).

26J. Zak, Phys. Rev. B 43, 4519 (1991); C. F. Hart and D. Emin, ibid. 43, 4521 (1991).

27J. Leo and A. MacKinnon, Phys. Rev. B 43, 5166 (1991); D. Emin and C. F. Hart, ibid. 43, 5169 (1991).

${ }^{28}$ L. Kleinman, Phys. Rev. B 41, 3857 (1990); D. Emin and C. F. Hart, ibid. 41, 3859 (1990).

${ }^{29}$ R. W. Koss and L. N. Lambert, Phys. Rev. B 5, 1479 (1972).

${ }^{30}$ E. E. Mendez, F. Agulló-Rueda, and J. M. Hong, Phys. Rev. Lett. 60, 2426 (1988).

${ }^{31}$ J. Bleuse, G. Bastard, and P. Voisin, Phys. Rev. Lett. 60, 220 (1988).

${ }^{32}$ F. Agulló-Rueda, E. E. Mendez, and J. M. Hong, Phys. Rev.
B 40, 1357 (1989).

33J. P. Hagon and M. Jaros, Phys. Rev. B 41, 2900 (1990).

${ }^{34}$ L. Esaki and R. Tsu, IBM J. Res. Dev. 14, 61 (1970).

${ }^{35}$ R. Tsu and G. Döhler, Phys. Rev. B 12, 680 (1975); G. Döhler, R. Tsu, and L. Esaki, Solid State Commun. 17, 317 (1975).

${ }^{36}$ F. Beltram, F. Capasso, D. L. Sivco, A. L. Hutchinson, S. N. G. Chu, and A. Y. Cho, Phys. Rev. Lett. 64, 3167 (1990).

${ }^{37}$ A. Sibille, J. F. Palmier, H. Wang, and F. Mollot, Phys. Rev. Lett. 64, 52 (1990).

${ }^{38}$ R. Tsu and L. Esaki, Phys. Rev. B 43, 5204 (1991).

${ }^{39}$ X. L. Lei, N. J. M. Horing, and H. L. Cui, Phys. Rev. Lett. 66, 3277 (1991).

${ }^{40}$ M. Nakayama, I. Tanaka, H. Nishimura, K. Kawashima, and K. Fujiwara, Phys. Rev. B 44, 5935 (1991); H. Schneider, K. Kawashima, and K. Fujiwara, ibid. 44, 5943 (1991). 